\title{
Contact problems between optical lenses and shrink fitter for a new type of laser microscope with a wide field of view
}

\author{
I. Nitta ${ }^{1} \&$ A. Kanno ${ }^{2}$ \\ ${ }^{1}$ Department of Mechanical and Production Engineering, \\ Niigata University, Japan \\ ${ }^{2}$ Sanjo Tsubame area Research Core, Japan
}

\begin{abstract}
We have studied contact stiffness between randomly rough surfaces. This research led to the development of a shrink fitter consisting of a new ring-shaped machine element to join mechanical components with different coefficients of thermal expansion, such as a ceramic shaft and a metal cylinder. As one application of the shrink fitter, several optical lenses were shrink-fitted in a lens-barrel. Such a scanning lens, assembled using the shrink fitter, can focus laser light well over a wide scanning width because the optical axes of the several lenses in the lens-barrel compliment each other well regardless of changes in room temperature. Thus, a laser microscope with such a scanning lens can observe object surfaces over a relatively wide area. However, contact pressures between the shrink fitter and the optical lenses will change the curvature of the lenses resulting in deterioration of the scanning properties of the scanning lens. Therefore, it is very important to calculate the contact pressures acting on optical lenses to examine the scanning properties of the scanning lens. In this study, we have developed a new type of laser microscope with a field of view of $10 \times 8 \mathrm{~mm}$. The pixel number in the laser scanning direction of $10 \mathrm{~mm}$ was 20,000 and 16,000 in the perpendicular direction of $8 \mathrm{~mm}$. Thus, one field of view of this laser microscope had 320,000,000 pixels. The observation results of some surfaces by this laser microscope are reported.
\end{abstract}

Keywords: contact pressure, shrink fitter, laser microscope, field of view, lens. 


\section{Introduction}

An optical or scanning electron microscope is usually used to observe object surfaces. However, only a very small fraction of the surface can be observed at a time because of limitations of the field of view of such microscopes, Nitta et al [1]. To obtain an image of the whole object surface to be investigated, large numbers of pictures of adjacent positions must be photographed using a CCD camera through the microscope with the specimen moved in a step-by-step feed operation. These pictures are combined with each other to make an image of the whole object surface. Thus, such observations have hardly been performed. However, observation of the whole surface will provide better information than that of only part of the surface. We have been engaged in attempts to make a new type of laser microscope with a resolution almost as high as that of optical microscopes and with a much wider field of view.

Figure 1 shows a schematic diagram of a new type of laser microscope, the key technology in which is a scanning lens unit, $\mathrm{f} \theta$ lens. It is necessary to converge a laser beam bundle on the object surface, i.e., the focal plane, to a few micrometers over a wide scanning width. Such a fine laser spot can be focused easily on the object surface only at the centre of the scanning width, but is difficult at the ends. The precise location of each scanning lens relative to the housing can adversely affect the size and shape of the laser spot on the focal plane, especially near the ends of the scanning width. The optical axes of each lens must be coincident with each other and should not be moved even with changes in room temperature, Yoder [2].

Here, we report that a shrink fitter, a new machine element developed by one of the authors, Nitta et al [3-9], greatly improves performance of the f $\theta$ scanning lens. The fitting pressure between the optical lenses and the metallic housing decreases with temperature because their coefficients of thermal expansion differ from each other. However, the shrink fitter could maintain the fitting pressure of the shrinkage fit of such a combination despite changes in room temperature.

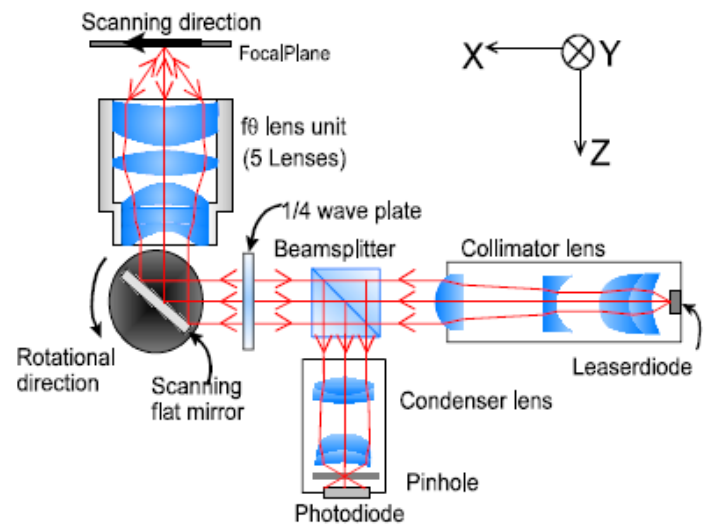

Figure 1: $\quad$ Schematic diagram of the new type of laser microscope. 
With this shrink fitter technology, we have developed a new type of laser microscope with a field of view of $10 \times 8 \mathrm{~mm}$. The pixel number in the laser scanning direction of $10 \mathrm{~mm}$ is 20,000 and that in the perpendicular direction of $8 \mathrm{~mm}$ is 16,000 . The observation results of some surfaces using this laser microscope are described.

\section{Shrink fitter}

The shrink fitter is a new machine element of cylindrical geometry. When two machine elements with different thermal expansion coefficients are shrink-fitted, the fitting pressure will vary with temperature. However, it is possible to keep the fitting pressure constant using the shrink fitter, even if the circumferential temperature changes. The shrink fitter has been applied to the connection of polygon mirror and self-acting air bearing, Nitta et al [7]. In addition, an assembly of an all-ceramic bearing and a metal housing has also been examined to improve the performance of the ceramic bearing using the shrink fitter. In the present study, the shrink fitter was applied to accurately locate the lenses in the housing of a new type of laser microscope with a wide field of view. The cylindrical shrink fitter is made of a plastic material, the Young's modulus of which is lower than that of the metal by about two orders of magnitude. This means there will be a larger degree of interference for the shrinkage fit of such a combination. If the room temperature increases, the housing will expand more than the lenses. The interference of the shrinkage fit, if the shrink fitter is not used, will decrease at elevated temperatures. However, the coefficient of thermal expansion of the plastic material is larger than that of the metal. Thus, if the thickness of the shrink fitter is designed appropriately, the interference will not change even at elevated temperature. Consequently, the contact pressure acting on the lens rim due to the shrinkage fit will be kept constant regardless of changes in temperature.

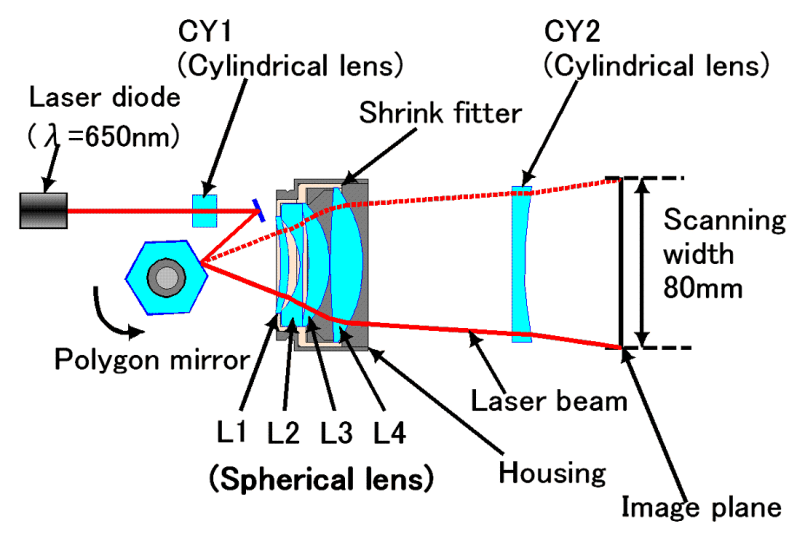

Figure 2: $\quad$ Laser scanner for description of lens deformations. 


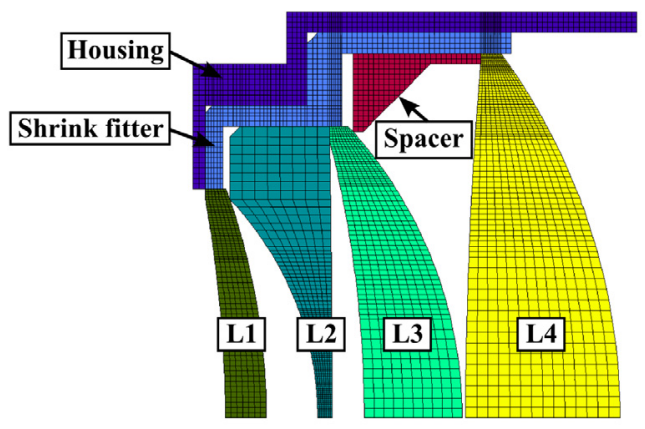

Figure 3: $\quad$ FEM mesh of the assembly.

\section{Shrink fitter method for a new laser microscope: procedure for critical interference}

In the shrink fit method, excessive interference will cause large-scale deformation of fitted lenses, resulting in deterioration of the focusing performance, Nitta et al [3]. Therefore, the critical interference under which the focusing performance does not become worse must be determined for appropriate use of the shrink fit method. A procedure to obtain the critical interferences is described below along with the laser scanner system shown in fig. 2. The f $\theta$ lens shown in fig. 2 consists of four lenses, named L1, L2, L3 and L4, respectively. These four lenses were shrink-fitted into an aluminium housing by the shrink fitter. A laser beam with a wavelength of $650 \mathrm{~nm}$ is irradiated from a laser diode. The $\mathrm{f} \theta$ lens was designed so that the laser spot may be kept at a constant diameter of $17 \mu \mathrm{m}$ over a wide scanning width of $80 \mathrm{~mm}$ on the image plane. Figure 3 shows two-dimensional FEM meshes of the $f \theta$ lens assembly. The mechanical properties of each component are shown in table 1 . Deformations of each lens corresponding to various interferences were calculated in an axisymmetric analysis using commercial FEM software, MARC, developed by MSC Software Corporation. The calculated lens deformations of both the entrance plane of L1 and the exit plane of L4 are shown together with the measured values in fig. 4. The calculated deformations were in excellent agreement with the measured values. The laser beam diameters were then simulated using commercial optical design software, CodeV, developed by Optical Research Associates, taking all lens deformations into consideration. Table 2 shows the critical interference of each lens under which the laser spot diameter on the image plane does not become larger than the designed value of $17 \mu \mathrm{m}$. The allowable interference is $60 \mu \mathrm{m}$. Thus, we can assemble the $\mathrm{f} \theta$ lens by the shrink fitter method without reducing the focusing performance if the interference can be kept below the critical value of $60 \mu \mathrm{m}$. In measurement of the sizes of the laser beam spots on the image plane shown in fig. 2, we confirmed that the laser beam bundles could be kept constant at about $17 \mu \mathrm{m}$ over the wide scanning width of $80 \mathrm{~mm}$ when the interferences were below $60 \mu \mathrm{m}$. 

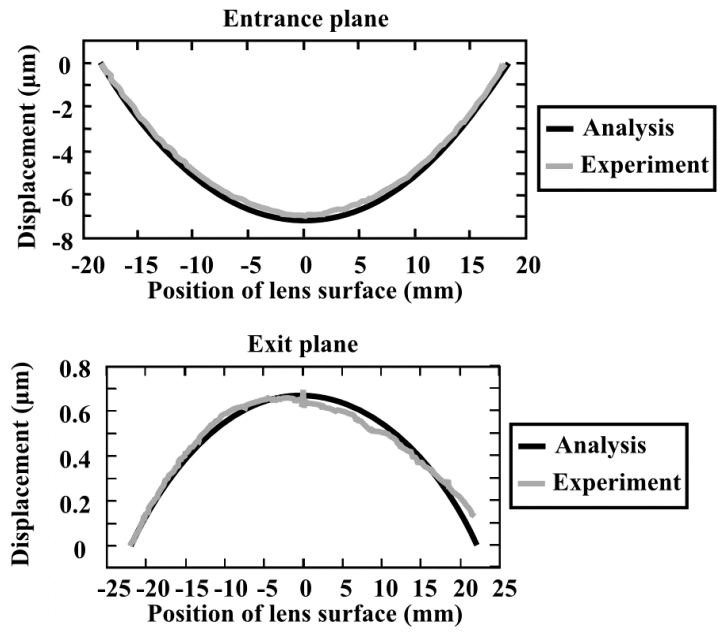

Figure 4: $\quad$ Profile of the deformed lens surface.

Table 1: $\quad$ Mechanical properties of each element.

\begin{tabular}{|c|c|c|c|}
\hline Elements & Materials & $\begin{array}{c}\text { Young's Modulus } \\
{[\mathrm{GPa}]}\end{array}$ & $\begin{array}{c}\text { Poisson's } \\
\text { Ratio }\end{array}$ \\
\hline L1 & Glass & 83.2 & 0.25 \\
\hline L2 & Glass & 55.2 & 0.23 \\
\hline L3 & Glass & 111.8 & 0.28 \\
\hline L4 & Glass & 106.1 & 0.28 \\
\hline Shrink fitter & POM & 2.8 & 0.47 \\
\hline Housing & Al(A5056B) & 69.0 & 0.34 \\
\hline
\end{tabular}

Table 2: $\quad$ Limited interference of each lens simulated by FEM and CODEV $[\mu \mathrm{m}]$.

\begin{tabular}{|c|c|c|c|c|}
\hline Lens No. & L1 & L2 & L3 & L4 \\
\hline Allowable value & 64 & 67 & 60 & 521 \\
\hline
\end{tabular}

\section{Outline of the developed laser microscope}

Figure 1 shows a schematic diagram of the new type of laser microscope developed in this study. First, a laser beam bundle emitted by a laser diode was made as parallel as possible through a collimating lens. In addition, a collimated laser beam, which was linearly polarised, was transformed into a circularly polarised beam by a quarter wave plate. The laser beam was scanned by a rotating flat mirror at a rotational speed of $9,000 \mathrm{rpm}$ and passed through the $\mathrm{f} \theta$ 
lens unit to focus the laser beam bundle on the focal plane at a laser spot diameter of about $3 \mu \mathrm{m}$ over a scanning width of $10 \mathrm{~mm}$. Then, via a polarising beam splitter and the quarter wave plate, the outgoing laser beam and the reflected laser beam became polarised at right angles to each other, which allowed good separation at the polarising beam splitter. Finally, the reflected laser beam passed to a photo-detector through a pinhole. The intensity of the reflected laser beam was transformed into digital data by a 12-bit A/D converter at a conversion rate of $100 \mathrm{MHz}$. The rotating flat mirror scanned the laser beam in the horizontal direction and the specimen attached to the motor-driven stage could be moved in the vertical direction at a constant speed controlled by a microcomputer. Thus, the surface of the specimen being observed could be scanned by the fine laser beam and the image of the surface be made by arranging the signals of the reflected laser light in the horizontal and vertical directions.

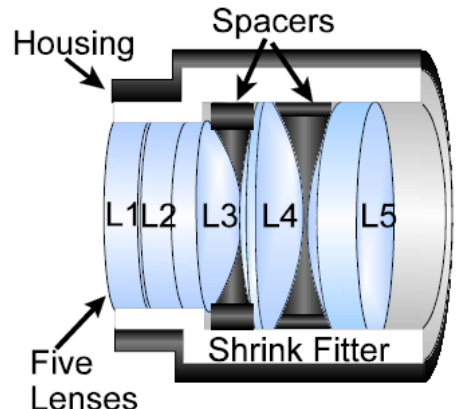

Figure 5: $\mathrm{f} \theta$ lens for the laser Figure 6:

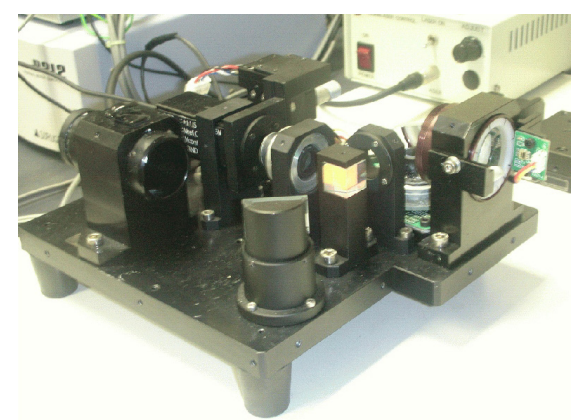

Developed microscope.

This $\mathrm{f} \theta$ lens unit has a telecentric property such that the outgoing laser beams from the $\mathrm{f} \theta$ lens to the specimen to be inspected are parallel to the optical axis of the $\mathrm{f} \theta$ lens unit. Telecentric lenses yield constant magnification over a range of working distances, virtually eliminating viewing angle error. With telecentric lenses, the image size remains almost unchanged when the object distance changes, provided the object to be inspected stays within the given field depth/telecentric range. In addition, the $\mathrm{f} \theta$ lens unit must be designed such that the image height is proportional to the scan angle (Theta), not the tangent of that angle: converting the equiangular motion of the laser beams to the constant speed motion for the scanning operation.

Figure 5 shows the $\mathrm{f} \theta$ lens unit used in this study, consisting of 5 lenses: named L1-L5 from the left side. The diameters of the lenses are $25 \mathrm{~mm}$ and 28 $\mathrm{mm}$. Figure 6 shows a photograph of the new type of laser microscope. 


\section{Basic performance of the laser microscope}

The laser spot diameters on the focal plane were simulated using the optical design software, Code V. The $\mathrm{f} \theta$ lens was designed so that the laser spot diameters could be kept at almost a constant value of $2 \mu \mathrm{m}$ over the whole scanning width. Figure 7 shows the laser spot diameters as a function of scanning position over the scanning width of $10 \mathrm{~mm}$, measured using a SpotScan model 0390, Photon Inc. The laser spot sizes at several scanning positions were measured in two different directions: one was in the scanning direction by the rotating flat mirror, main scanning direction, and the other was in the orthogonal direction, sub- (or vice-) scanning direction, as the laser spot emitted from the laser diode is not perfectly circular but slightly elliptical although the laser beam from the laser diode is reshaped into an almost circular spot through two cylindrical lenses before the collimator lens. The laser spots measured at the middle of the scanning width in the main scanning direction were about $2.7 \mu \mathrm{m}$ in diameter. The laser spots became larger as the laser beam was scanned toward both the scanning ends. If the diameters of the laser spot in two different directions are compared, the spot diameter in the main scanning direction is smaller than that in the sub-scanning direction.

The resolution of the laser microscope was evaluated using a USAF resolution target, consisting of bars organised into groups and elements, Edmund Optics [10]. A positive target has only the pattern chrome deposited on the glass substrate, so it had a black pattern with a clear field. Each group consists of six elements (i.e., elements 1-6), each of which is composed of three horizontal and three vertical equally spaced bars. Each element within a group corresponds to an associated resolution, based on the bar width/space, in the range from $0.8 \mu \mathrm{m}$ to $500 \mu \mathrm{m}$. The group and element define the resolution of an imaging system just before the black and white bars begin to blend together. The vertical bars are used to calculate horizontal resolution and the horizontal bars are used to calculate vertical resolution. One line pair equals one black and one white bar.

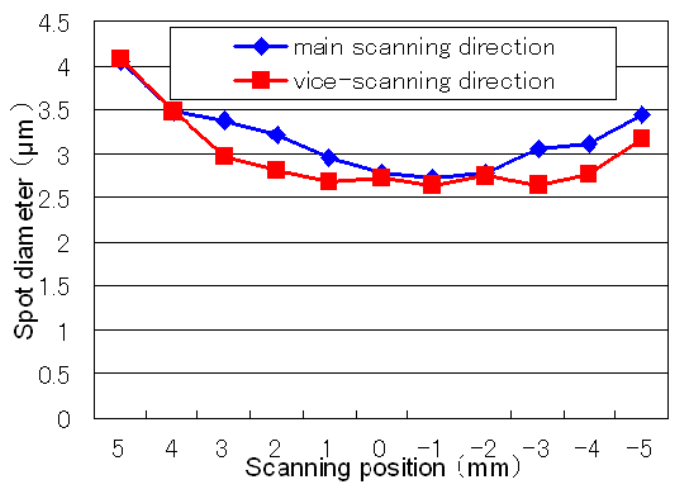

Figure 7: Measured laser spot sizes. 


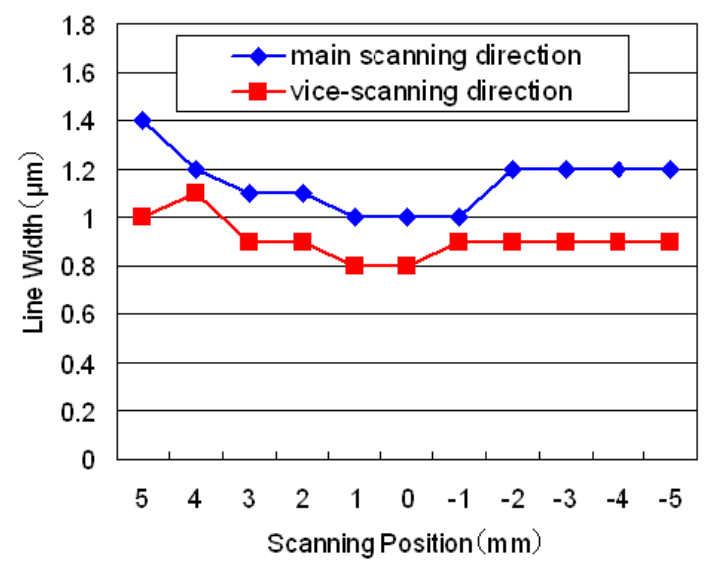

Figure 8: $\quad$ Measured resolution, MTF.

Figure 8 shows the line widths of the resolution targets that can be resolved by the laser microscope as a function of the scanning position. The resolution in the main scanning direction was about $1 \mu \mathrm{m}$ around the middle of the scanning width. It decreased towards both the scanning ends corresponding to the tendency of the laser spot sizes. The resolutions in the sub-scanning direction were slightly better than those in the main scanning direction corresponding to the laser spot sizes on the focal plane shown in fig. 7.

On the other hand, the same resolution target was also observed with an ordinary optical microscope. Using an objective with a magnification of 10 the resolution was about $1.0 \mu \mathrm{m}$. Thus, the magnification of the laser microscope was about 10 by conversion of the objective of the optical microscope.

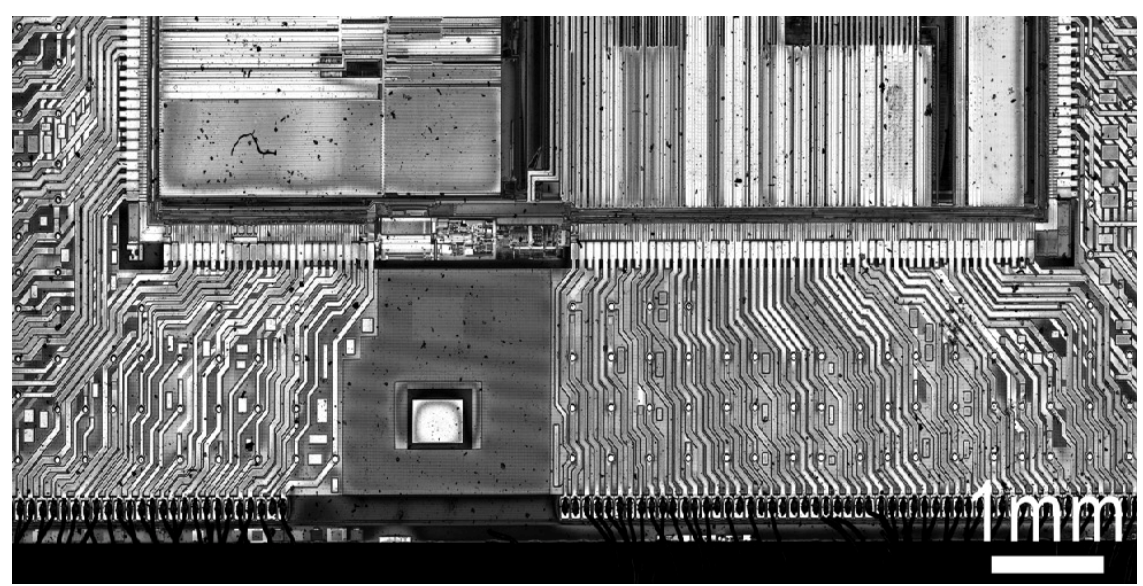

Figure 9: $\quad$ Image of a CPU observed using the developed laser microscope. 


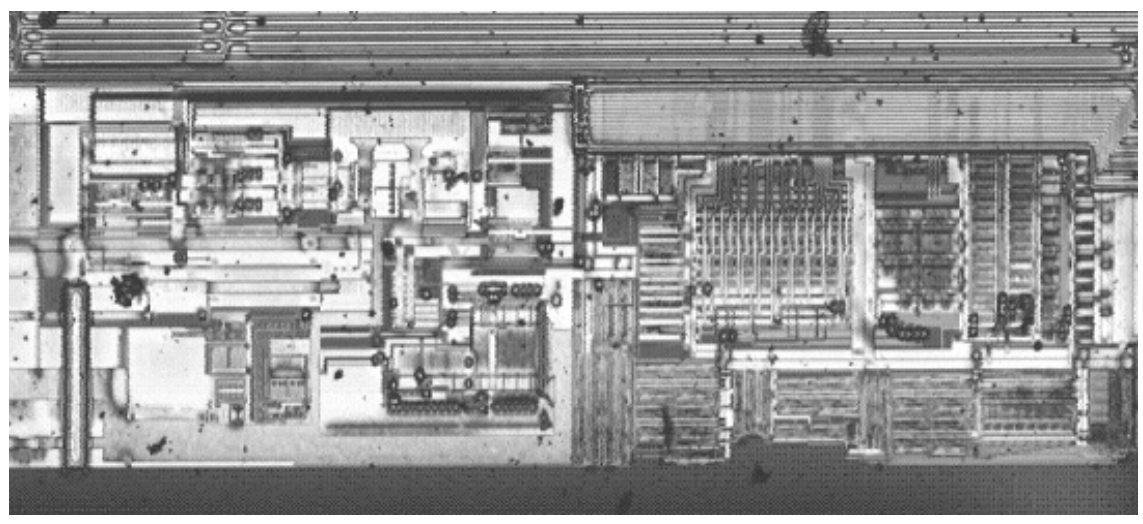

Figure 10: Detailed image of the white rectangular portion in fig. 9.

\section{Images observed with the laser microscope}

Figure 9 shows an image of a CPU observed with the laser microscope. The cover of the CPU package for a personal computer was removed and set on the stage of the laser microscope to observe its circuit pattern. The measured area was $10 \mathrm{~mm}$ in width and $4 \mathrm{~mm}$ in height. The scanning width of $10 \mathrm{~mm}$ is a limitation of the $\mathrm{f} \theta$ lens unit. However, the vertical scanning width of $4 \mathrm{~mm}$ is not a limitation of the laser microscope but is dependent on the limitation of the mechanical stage used in the laser microscope. Thus, longer surfaces than this can also be observed.

It is obvious that a huge area can be observed at a time, compared with the conventional optical microscope. This laser microscope also has a deep depth-of-focus so that the image was very clear despite the rough surface.

The pixel number in the laser scanning direction of $10 \mathrm{~mm}$ was 20,000 , and thus the distance from pixel to pixel is $0.5 \mu \mathrm{m}$. Figure 9 has a total of $320,000,000$ pixels. Thus, any portion in fig. 9 can be seen at higher magnification. Figure 10 shows a portion of fig. 9 surrounded by the white rectangle on a finer scale; very fine patterns that could not be seen in fig. 9 were visible.

\section{Conclusions}

The shrink fitter technology has made it possible to shrink fit optical lenses into the housing without serious reduction of the focusing performance. The critical interferences under which the focusing performance does not become worse were obtained. The procedure to obtain the critical interferences was described. With the shrink fitter technology, a new type of laser microscope with a huge field of view was developed. Several images obtained using this laser microscope were presented. 


\section{References}

[1] Nitta, I., Kanno, A., Komata,K. \& Iguchi, S., "New joining method for laser scanner lenses by using a shrink fitter", Proc. $5^{\text {th }}$ int. conf. on computational methods in contact mechanics, ed. Dominguez \& Brebbia C.A., WIT press, pp.31-40, 2001.

[2] Yoder, P.R., "Opto-mechanical systems design, third edition”, CRC Press, pp.202-204, 2005.

[3] Nitta, I., Kanno, A. \& Komata,K., "Effect of Interference on Scanning Performance of $\mathrm{f} \theta$ Lens Fixed by a Shrink Fitter", Optical review, Vol.10, No.4, pp.321-324, 2003.

[4] Nitta, I., Kigoshi, K., \& Kato, K., "Study of the fitting strength between ceramic and metal elements with the use of a shrink fitter at elevated temperature", JSME international Journal, Series III, 32, pp.632-639, 1989 ,

[5] Nitta, I., Nakashizuka, K. \& Hara, T., "The fitting strength between ceramic and metal with the use of a bimetal shrink fitter at elevated temperature", JSME international Journal, Series I, 34, pp.249-256, 1991.

[6] Nitta, I., Kusama, K. \& Hara, T., “ Shrink fit between a ceramic and a metal element using a hybrid shrink fitter, JSME international Journal, Series C, 38, pp.617-624, 1995.

[7] Nitta, I., Furukawa, H., Komata, K. \& Konno, D., "New method of joining a polygon mirror using a shrink fitter (in Japanese), Trans. Jpn. Soc. Mech. Eng. 62, pp.2785-2791, 1996.

[8] Nitta, I., Kanno, A., Komata,K. \& Iguchi, S., "New joining method for laser scanner lenses by using a shrink fitter", Proc. $5^{\text {th }}$ int. conf. on computational methods in contact mechanics, ed. Dominguez \& Brebbia C.A., WIT press, pp.31-40, 2001.

[9] Nonaka, S., Nitta, I., Kanno, A. \& Nishimura, M., "Study of a laser material processing system with fine optical setup using a shrink fitter", Proc. of the Int. Conf. Leading Edge Manufacturing in $21^{\text {st }}$ Century, pp.873-877, 2003.

[10] EdmundOptics, http://www.edmundoptics.com/techSupport/Display Article.cfm/articleid $=248$. 